\title{
Comparative role of various methods of estimating between study variance for meta-analysis using random effect method
}

\author{
Mona Pathak $^{\mathrm{a}, \mathrm{b}}$, Sada Nand Dwivedi ${ }^{\mathrm{b}, *}$, Bhaskar Thakur ${ }^{\mathrm{a}}$

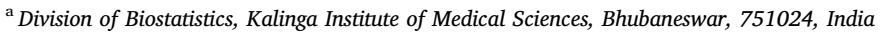 \\ ${ }^{\mathrm{b}}$ Department of Biostatistics, All India Institute of Medical Sciences, New Delhi, 110029, India
}

\section{A R T I C L E I N F O}

\section{Keywords:}

DerSimonnian \& Laird method

Coverage probability

Meta-analysis

Hazard ratio

Random effect method

\begin{abstract}
A B S T R A C T
Methods of synthesizing the effect size is guided by observed heterogeneity. As a convention, fixed effect method (FEM) is used for low/no heterogeneity. However, random effect method (REM) is used for substantial heterogeneity. But, synthesized (i.e., pooled) effect size under REM also relies on the method used to estimate between study variance along with within study variance. There are various methods to assess between study variance to be used under REM. Accordingly, present study compared existing methods of estimating between study variance on the basis of coverage probability and precision in order to find preferred method of assessing between study variance. Data from a systematic review and meta-analysis for various outcomes involving varying extent of sample size and heterogeneity was used. Hunter and Schmidt method and DerSimonnian \& Laird method were found as preferred methods to estimate between study variance.
\end{abstract}

\section{Introduction}

Meta-analysis involves statistical techniques to synthesize the effect size of the considered outcome from various comparative studies. ${ }^{1}$ Comparative studies too have some degree of heterogeneity because of variation in population, intervention, comparator and outcome characteristics. Further, choice of meta-analysis method depends on prevailing heterogeneity. As a convention, fixed effect method (FEM) is used in case of no heterogeneity as it assumes that all the studies are measuring a single true effect size and variation in the study level effect size is due to sampling alone. But, fixed effect method assumes that between studies variation is zero. On the other hand, random effect method (REM) assumes that study level effect size is a random sample out of the infinite studies following the same inclusion criteria for metaanalysis. Pooled effect estimate under fixed effect method is weighted average of study level effect sizes considering within study variance as weights. Also, instead of considering within study variance, other forms of weights can also be considered like sample size, study quality or a function of such parameters. On the other hand, REM accounts for the between study heterogeneity considering both within as well as between study variance. However, various methods exist to assess the between study variance. Accordingly, the pooled effect estimate may rely on the estimated between study variance. Therefore, present study compared the performance of various methods to estimate between study variance under meta-analysis using random effect method(REM).

\section{Materials \& methods}

\subsection{Data}

For the present article, data of a systematic review of randomized controlled trials comparing neoadjuvant chemotherapy with adjuvant chemotherapy for oncological and functional outcomes were considered. The detail methodology, definition of outcomes and result of the systematic review is published elsewhere. ${ }^{2}$

\subsection{Random effect method}

The individual study effect size under random effect method can be modeled as:

$y_{i}=\mu+\theta_{i}+\varepsilon_{i}$

Where $\theta_{i}$ is deviation between individual level study effect size $\left(y_{\mathrm{i}}\right)$ and the true effect $(\mu)$ which exceeds due to sampling variation $\left(\varepsilon_{i}\right)$ alone. Further, $\theta_{i}$ depends on between study variance $\left(\tau^{2}\right)$ while $\varepsilon_{i}$ depends on the within study variance $\left(\sigma_{i}^{2}\right)$ alone. Hence $y_{i}$ is distributed as $y_{i} \sim N\left(\mu, \sigma_{i}^{2}+\tau^{2}\right)$.

As random effect method considers random sample of the effect sizes, the mean of the deviation in effect size will obviously be zero. As

\footnotetext{
* Corresponding author. Department of Biostatistics, All India Institute of Medical Sciences, Ansari Nagar, New Delhi, 110029, India.

E-mail addresses: dwivedi7@hotmail.com,dwivedi7@gmail.com,dwivedi7@aiims.edu, dwivedi@aiims.ac.in (S.N. Dwivedi).
} 
such, random effect method assumes that $\theta_{i}$ is distributed normally with mean zero and variance $\left(\tau^{2}\right)$ as $\theta_{i} \sim N\left(0, \tau^{2}\right)$, and sampling error is also distributed normally as $\varepsilon_{i} \sim N\left(0, \sigma_{i}^{2}\right)$. Since random effect method considers two sources of variation, between study variance as well as within study variance, the weights $\left(w_{i}\right)$ associated with individual studies are inverse of this total variation:

$w_{i}=1 /\left(\sigma_{i}^{2}+\tau^{2}\right)$

The pooled effect estimate of $y_{i}$ may be given as below:

$\hat{\mu}=\sum y_{i} w_{i} / \sum w_{i}$

Further, the variance of this estimated effect size may be represented as:

$V(\hat{\mu})=1 / \sum w_{i}$

The random effect method rely depend on the estimated value of between study variance. This between study variance i.e., $\tau^{2}$, may however be estimated using various methods.

The methods to estimate between study variance are categorized in two parts as non-iterative methods and iterative methods. ${ }^{3}$ Non-iterative methods of variance estimation are formula based methods which do not require iterative algorithm. On the other hand, iterative methods may not converse always. Hence, present study chose to compare noniterative methods of estimating variance. These methods are given as follows:

\subsection{Method of moments ${ }^{4}$}

In case of $\mathrm{k}$ studies, let us assume that $s_{1}^{2}, \ldots \ldots ., s_{k}^{2}$ and $t^{2}$ are respective estimates of within study variances $\left(\sigma_{1}^{2}, \ldots \ldots, \sigma_{k}^{2}\right)$ and between study variance $\left(\tau^{2}\right)$. Estimate of pooled effect estimate is $\overline{\mathrm{T}}=\frac{\sum_{i} m_{i} T_{i}}{\sum_{i} m_{i}}$, where $m_{1}, \ldots \ldots m_{k}$ are study level considered weights (any positive constants). For variance estimation, the expected value of the expression $\mathrm{Q}=\sum_{i} m_{i}\left(T_{i}-\bar{T}\right)^{2}$ is:

$E\left[\sum_{i} m_{i}\left(T_{i}-\bar{T}\right)^{2}\right]=\sum_{i} m_{i}\left(\tau^{2}+\sigma_{i}^{2}\right)-\sum_{i} m_{i}\left(\tau^{2}+\sigma_{i}^{2}\right) / \sum_{i} m_{i}$

This can be expressed as:

$$
\begin{aligned}
\mathrm{E}\left[\sum_{\mathrm{i}} \mathrm{m}_{\mathrm{i}}\left(\mathrm{T}_{\mathrm{i}}-\overline{\mathrm{T}}\right)^{2}\right]= & \tau^{2}\left[\sum_{\mathrm{i}} \mathrm{m}_{\mathrm{i}}-\sum_{\mathrm{i}} \mathrm{m}_{\mathrm{i}}^{2} / \sum_{\mathrm{i}} \mathrm{m}_{\mathrm{i}}\right] \\
& +\left[\sum_{\mathrm{i}} \mathrm{m}_{\mathrm{i}} \sigma_{\mathrm{i}}^{2}-\sum_{\mathrm{i}} \mathrm{m}_{\mathrm{i}} \sigma_{\mathrm{i}}^{2} / \sum_{\mathrm{i}} \mathrm{m}_{\mathrm{i}}\right]
\end{aligned}
$$

By equating the $\sum_{\mathrm{i}} \mathrm{m}_{\mathrm{i}}\left(\mathrm{T}_{\mathrm{i}}-\overline{\mathrm{T}}\right)^{2}$ to its expected value in equation (6), and substituting $\mathrm{s}_{1}^{2}, \ldots \ldots . ., \mathrm{s}_{\mathrm{k}}^{2}$ for $\sigma_{1}^{2}, \ldots \ldots, \sigma_{\mathrm{k}}^{2}$, the value of $\tau^{2}$ using method of moments (MM) can be estimated ${ }^{4,5}$ :

$\tau^{2}(M M)=\frac{\left[\sum_{i} m_{i}\left(T_{i}-\bar{T}\right)^{2}\right]-\left[\sum_{i} m_{i} s_{i}^{2} / \sum_{i} m_{i}\right]}{\left[\sum_{i} m_{i}-\frac{\sum_{i} m_{i}^{2}}{\sum_{i} m_{i}}\right]}$

In case, if $\tau^{2}(M M)$ is negative, it is truncated at zero. Most of the methods to estimate $\tau^{2}$ were derived from the method of moments by specifying varying values of weight $m_{i}$.

\subsection{Cochrane ANOVA method ${ }^{5,6}$}

Cochrane ANOVA method assumes that each study provides equal information and is of equal size. ${ }^{5,6}$ Accordingly, by substituting $a_{i}=\frac{1}{k}$ in equation (7) yields the Cochrane ANOVA (CA) estimate of $\tau^{2}$.

$\tau^{2}(C A)=\max \left\{0, \frac{1}{k-1} \sum_{i}\left(T_{i}-\bar{T}_{C A}\right)^{2}-\frac{1}{k} \sum_{i} s_{i}^{2}\right.$
$\bar{T}_{C A}=\frac{\sum_{i} T_{i}}{k}$

\subsection{DerSimonian and Liard method ${ }^{7}$}

DerSimonian and Liard (1986) proposed another method to estimate $\tau^{2}$ by taking precision of each study as weight. Under this method, substituting $a_{i}=\frac{1}{s_{i}^{2}}$ in equation (7) yields the DerSimonian and Liard estimate (DL) of $\tau^{2}$.

$\tau^{2}(D L)=\max \left\{0, \frac{\left[\sum_{i} w_{i D L}\left(T_{i}-\overline{\mathrm{T}}_{D L}\right)^{2}\right]-(k-1)}{\left[\sum_{i} w_{i D L}-\frac{\sum_{i} w_{i D L}^{2}}{\sum_{i} w_{i D L}}\right]}\right.$

$\bar{T}_{D L}=\frac{\sum_{i} w_{i D L} T_{i}}{\sum_{i} w_{i D L}}$ and $w_{i D L}=1 / s_{i}^{2}$

DerSimonian and Liard method is most common method of used under random effect analysis. So it is widely available in the software facilitating meta-analysis.

\subsection{Two step DerSimonian and Liard method ${ }^{5}$}

DerSimonain and Kacker ${ }^{5}$ proposed a two steps estimator. It is also based on the method of moment estimator by substituting $\tau^{2}$ with $\tau_{D L}^{2}$ in equation $m_{i}=\frac{1}{s_{i}^{2}+\tau_{D L}^{2}}$ and with $\bar{T}$ with $\bar{\mu}_{D L}$ (Pooled effect estimate under DerSimonian \& Liard method) in equation (7). The two steps DerSimonian and Laird Method (DL2) of between study variance can be calculated as:

$$
\begin{aligned}
& \tau^{2}(\mathrm{D} L 2) \\
& =\left\{0, \frac{\left[\sum_{i} w_{i D L 2}\left(T_{i}-\bar{\mu}_{D L}\right)^{2}\right]-\left[\sum_{i} w_{i D L 2} s_{i}^{2}-\sum_{i} w_{i D L 2}^{2} s_{i}^{2} / \sum_{i} w_{i D L 2}\right]}{\left[\sum_{i} w_{i D L 2}-\frac{\sum_{i} w_{i D L 2}^{2}}{\sum_{i} w_{i D L 2}}\right]}\right.
\end{aligned}
$$

Where

$w_{i D L 2}=1 /\left(\tau^{2}(D L)+s_{i}^{2}\right)$

\subsection{Two step cochrane ANOVA method ${ }^{5}$}

DerSimonain and Kacker ${ }^{5}$ also proposed another two steps estimator. In first step $\tau_{C A}^{2}$ and $\bar{\mu}_{C A}$ is estimated by using Cochrane ANOVA methods. In the second step, between study variance using two step Cochrane ANOVA method (CA2) is estimated by substituting $m_{i}=\frac{1}{s_{i}^{2}+\tau_{C A}^{2}}$ and $\bar{T}$ with $\bar{T}_{C A}$ in equation (7).

$$
\begin{aligned}
& \tau^{2}(C A 2) \\
& =\left\{0, \frac{\left[\sum_{i} w_{i C A 2}\left(T_{i}-\bar{\mu}_{C A}\right)^{2}\right]-\left[\sum_{i} w_{i C A 2} s_{i}^{2}-\sum_{i} w_{i C A 2}^{2} S_{i}^{2} / \sum_{i} w_{i C A 2}\right]}{\left[\sum_{i} w_{i C A 2}-\frac{\sum_{i} w_{i C A 2}^{2}}{\sum_{i} w_{i C A 2}}\right]} a\right.
\end{aligned}
$$

Where,

$w_{i C A 2}=1 /\left(\tau^{2}(C A)+s_{i}^{2}\right)$

\subsection{Hartung and Makambi Method ${ }^{8,9}$}

The method proposed by Hartung and Makambi ${ }^{8,9}$ is a modification of the method proposed by DerSimonnian and Liard method. ${ }^{7}$ Unlike other methods to estimate between study variance, it does not require truncation to zero. 
Table 1

Estimated Between Study Variance using Each of Variance Estimation Methods.

\begin{tabular}{|c|c|c|c|c|c|c|c|}
\hline Method & $\begin{array}{l}\text { Overall } \\
\text { Survival }\end{array}$ & $\begin{array}{l}\text { Disease Free } \\
\text { Survival }\end{array}$ & $\begin{array}{l}\text { Relapse Free } \\
\text { Survival }\end{array}$ & $\begin{array}{l}\text { Loco-Regional } \\
\text { Recurrence }\end{array}$ & $\begin{array}{l}\text { Local } \\
\text { Recurrence }\end{array}$ & $\begin{array}{l}\text { Distant } \\
\text { Metastasis }\end{array}$ & $\begin{array}{l}\text { Breast } \\
\text { Conserving } \\
\text { Surgery }\end{array}$ \\
\hline No of RCTs & 15 & 6 & 14 & 15 & 10 & 13 & 9 \\
\hline $\mathrm{I}^{2}$ Statistics & $0.0 \%$ & $26.3 \%$ & $47.2 \%$ & $0.0 \%$ & $0.0 \%$ & $43.5 \%$ & $90.1 \%$ \\
\hline DerSimonian \& Liard & 0.0000 & 0.0121 & 0.0336 & 0.0000 & 0.0000 & 0.0345 & 0.0439 \\
\hline Two Step DerSimonian \& Liard & 0.0000 & 0.0173 & 0.0677 & 0.0000 & 0.0000 & 0.0562 & 0.1339 \\
\hline Cochrane ANOVA & 0.0000 & 0.0910 & 0.1883 & 0.0000 & 0.0000 & 0.1627 & 2.3130 \\
\hline Two step Cochrane ANOVA & 0.0000 & 0.0396 & 0.1260 & 0.0000 & 0.0000 & 0.0910 & 1.4804 \\
\hline Hartung \& Makambi & 0.0108 & 0.0185 & 0.0346 & 0.0279 & 0.0226 & 0.0372 & 0.0408 \\
\hline Hunter and Schmidt & 0.0000 & 0.0025 & 0.0249 & 0.0000 & 0.0000 & 0.0242 & 0.0358 \\
\hline Sidik \& Jonkman & 0.0393 & 0.1072 & 0.1789 & 0.0872 & 0.0390 & 0.1617 & 1.4388 \\
\hline
\end{tabular}

$\frac{\sum w_{i, F}\left(T_{i}-\hat{\mu}_{F}\right)^{2}}{\sum w_{i, F}-\frac{\sum w_{i, F}^{2}}{\sum T_{i, F}}}=\frac{Q}{\sum w_{i, F}-\frac{\sum w_{i, F}^{2}}{\sum w_{i, F}}}$

where $w_{i, F}$ is weights under fixed effect method, i.e., inverse of within study variance only; $y_{i}$ and $\hat{\mu}_{F}$ are individual study effect size and pooled effect estimate (weighted average) under fixed effect method respectively. Under Hartung \& Makambi method, the quadratic form above is multiplied by the factor $Q /(2(k-1)+Q)$ to ensure positivity. ${ }^{3}$ The estimator is given as:

$\hat{\tau}_{H M}^{2}=\frac{Q^{2}}{2(k-1)+Q)\left(\sum w_{i, F}-\frac{\sum w_{i, F}^{2}}{\sum w_{i, F}}\right)}$

\subsection{Hunter and Schmidt Method ${ }^{10}$}

Another method proposed by Hunter and Schmidt ${ }^{10}$ is given by

$\hat{\tau}_{H S}^{2}=\left\{0, \frac{Q-k}{\sum w_{i, F}}\right\}$

\subsection{Sidik and Jonkman method ${ }^{11,12}$}

To calculate between study variance by Sidik and Jonkman $\operatorname{method}^{11,12}$, initial estimator of between study variance is calculated as $\hat{\tau}_{0}^{2}=\sum_{i}\left(T_{i}-\bar{T}\right)^{2} / k$. Then estimate of between study variance using Sidik and Jonkman method (SJ) may be obtained as:

$\hat{\tau}_{S J}^{2}=\frac{1}{k-1} \sum \mathrm{w}_{\mathrm{iSJ}}\left(T_{i}-\hat{T}_{\mathrm{SJ}}\right)^{2}$

Where

$\hat{T}_{S J}=\sum \mathrm{w}_{\mathrm{iSJ}} y_{i} / \sum \mathrm{w}_{\mathrm{iSJ}}$

Where

$\mathrm{w}_{\mathrm{iSJ}}=\frac{\hat{\tau}_{0}^{2}}{\mathrm{~s}_{\mathrm{i}}^{2}+\hat{\tau}_{0}^{2}}$

All the above discussed methods was used to estimate the between study variance $\left(\tau^{2}\right)$. The estimated value of between study variance was used to calculate the total variance, i.e., sum of within as well as between study variance. Pooled effect estimate under random effects method is weighted average of study level effect sizes considering this total variance as weight.

\subsection{Comparison of meta-analysis methods}

For each of the considered outcomes, the performance of random effects method using each of the method to estimate between study variance was compared using the following statistics:

\subsection{Coverage probability}

It measures the actual probability of the true value being included in the $95 \% \mathrm{CI}$ of pooled effect estimate. It is measured as proportion of how many times the confidence interval contains the true population parameter. The coverage probability of interval estimate synthesized by random effects method using a particular method of between study variance was calculated using 10000 bootstrap samples of individual studies. The pooled effect estimate by random effect method with that particular method of estimating between study variance was calculated for each of these 10000 samples. Further, the proportion of these effect estimates under each of the bootstrap sample lying within the $95 \% \mathrm{CI}$ of $\mu$ was considered as coverage probability.

\subsection{Width of confidence interval}

Along with coverage probability, the width of confidence interval also matters, so width of the confidence interval of pooled effect estimates was also considered while comparing difference variance estimation methods.

\section{Results}

Present study involved 18 RCTs comparing at least one of the considered outcomes. Various outcomes involved variable sample size, and variable extent of heterogeneity. The existing non-iterative methods of calculating between study variance were used to estimate between study variance for each of the outcomes. The estimates of between study variance $\left(\tau^{2}\right)$ by various methods are listed in Table 1 and pooled hazard ratio for all the outcomes except breast conserving surgery, for which it is risk ratio, are listed in Table 2 calculated by each of the considered methods. All methods estimated $\tau^{2}=0$ in case of no heterogeneity, i.e., $\mathrm{I}^{2}=0 \%$, in case of overall survival, local recurrence and loco-regional recurrence, except Hartung \& Makambi Method and Sidik \& Jonkman method. These two methods overestimated the $\tau^{2}$. As evident from Table 2, in case of no heterogeneity, all the methods gave exactly same results except in case of Hartung \& Makambi method and Sidik \& Jonkman method. These two methods overestimated the $\tau^{2}$ and hence lead to wider confidence interval of effect size. With increasing heterogeneity, the magnitude of difference between the estimated values of $\tau^{2}$ by different methods also increased. With very high heterogeneity like in case of breast conserving surgery, differences in estimated value of $\tau^{2}$ also increased from 0.0358 by Hunter \& Schmidt method to 2.313 by Cochrane ANOVA method.

As obvious, the width of confidence interval increases with increased heterogeneity as evident from case of breast conserving surgery. Random effect method using Hunter and Schmidt, and DerSimonnian \& Laird method of estimating between study variance had most precise confidence intervals in comparison to other methods.

Variance estimation methods were also compared on the basis of coverage probability of interval estimate of hazard ratio calculated 
Table 2

Hazard Ratio/Risk Ratio for Various Outcomes using Random Effect Method with Varying Between Study Variance Estimation Methods.

\begin{tabular}{|c|c|c|c|c|c|c|c|}
\hline Outcome & $\begin{array}{l}\text { Overall } \\
\text { Survival }\end{array}$ & $\begin{array}{l}\text { Disease free } \\
\text { Survival }\end{array}$ & $\begin{array}{l}\text { Relapse Free } \\
\text { Survival }\end{array}$ & $\begin{array}{l}\text { Loco-Regional } \\
\text { Recurrence }\end{array}$ & $\begin{array}{l}\text { Local } \\
\text { Recurrence }\end{array}$ & $\begin{array}{l}\text { Distant } \\
\text { Metastasis }\end{array}$ & $\begin{array}{l}\text { Breast } \\
\text { Conserving } \\
\text { Surgery }^{\mathrm{a}}\end{array}$ \\
\hline No of RCTs & 15 & 6 & 14 & 15 & 10 & 13 & 9 \\
\hline $\mathrm{I}^{2}$ Statistics & $0.0 \%$ & $26.3 \%$ & $47.2 \%$ & $0.0 \%$ & $0.0 \%$ & $43.5 \%$ & $90.1 \%$ \\
\hline DerSimonian \& Liard & $0.98(0.89-1.08)$ & $0.99(0.83-1.19)$ & $1.01(0.86-1.18)$ & $1.23(1.06-1.43)$ & $1.31(1.11-1.56)$ & $0.97(0.82-1.16)$ & $1.21(1.04-1.41)$ \\
\hline Two Step DerSimonian \& Liard & $0.98(0.89-1.08)$ & $0.99(0.82-1.21)$ & $0.98(0.81-1.19)$ & $1.23(1.06-1.43)$ & $1.31(1.11-1.56)$ & $0.96(0.79-1.17)$ & $1.24(0.97-1.60)$ \\
\hline Cochrane ANOVA & $0.98(0.89-1.08)$ & $0.98(0.70-1.37)$ & $0.93(0.70-1.23)$ & $1.23(1.06-1.43)$ & $1.31(1.11-1.56)$ & $0.93(0.71-1.23)$ & $1.59(0.60-4.18)$ \\
\hline Two step Cochrane ANOVA & $0.98(0.89-1.08)$ & $0.99(0.77-1.28)$ & $0.95(0.75-1.21)$ & $1.23(1.06-1.43)$ & $1.31(1.11-1.56)$ & $0.95(0.76-1.19)$ & $1.50(0.69-3.28)$ \\
\hline Hartung \& Makambi & $0.97(0.86-1.10)$ & $0.99(0.81-1.22)$ & $1.01(0.86-1.18)$ & $1.24(1.02-1.51)$ & $1.32(1.08-1.62)$ & $0.97(0.81-1.16)$ & $1.21(1.04-1.40)$ \\
\hline Hunter and Schmidt & $0.98(0.89-1.08)$ & $0.99(0.86-1.12)$ & $1.02(0.88-1.18)$ & $1.23(1.06-1.43)$ & $1.31(1.11-1.56)$ & $0.98(0.83-1.15)$ & $1.20(1.05-1.38)$ \\
\hline Sidik \& Jonkman & $0.96(0.81-1.13)$ & $0.97(0.68-1.39)$ & $0.93(0.71-1.23)$ & $1.23(0.96-1.57)$ & $1.32(1.06-1.65)$ & $0.93(0.71-1.23)$ & $1.50(0.69-3.23)$ \\
\hline
\end{tabular}

a For Breast Conserving Surgery, effect size is Risk Ratio.

Table 3

Coverage probability of pooled effect interval estimate calculated using random effect method with various variance estimation methods.

\begin{tabular}{|c|c|c|c|c|c|c|c|}
\hline Method & Overall Survival & $\begin{array}{l}\text { Disease Free } \\
\text { Survival }\end{array}$ & $\begin{array}{l}\text { Relapse Free } \\
\text { Survival }\end{array}$ & $\begin{array}{l}\text { Loco Regional } \\
\text { Recurrence }\end{array}$ & $\begin{array}{l}\text { Local } \\
\text { Recurrence }\end{array}$ & $\begin{array}{l}\text { Distant } \\
\text { Metastasis }\end{array}$ & $\begin{array}{l}\text { Breast } \\
\text { Conservation }\end{array}$ \\
\hline DerSimonian \& Liard & 0.9446 & 0.9246 & 0.9302 & 0.9489 & 0.9626 & 0.9460 & 0.8924 \\
\hline Two Step DerSimonian \& Liard & 0.9410 & 0.9191 & 0.9321 & 0.9492 & 0.9626 & 0.9536 & 0.9279 \\
\hline Cochrane ANOVA & 0.9600 & 0.9852 & 0.9855 & 0.9518 & 0.9626 & 0.9934 & 0.9786 \\
\hline Two step Cochrane ANOVA & 0.9413 & 0.9332 & 0.9589 & 0.9499 & 0.9626 & 0.9724 & 0.9284 \\
\hline Hartung \& Makambi & 0.9700 & 0.9319 & 0.9339 & 0.9815 & 0.9744 & 0.9585 & 0.8822 \\
\hline Hunter and Schmidt & 0.9529 & 0.8683 & 0.9220 & 0.9480 & 0.9626 & 0.9391 & 0.8645 \\
\hline Sidik \& Jonkman & 0.9903 & 0.9795 & 0.9740 & 0.9972 & 0.9830 & 0.9865 & 0.9284 \\
\hline
\end{tabular}

under random effect method using various between study variance estimation methods. The coverage probabilities under each of the considered methods for each of the considered outcomes are listed in Table 3. In the case of no heterogeneity, as obvious, the point as well as interval estimates under each method coincided except two methods, i.e., Hartung \& Makambi method and Sidik \& Jonkman method, where between studies variance was overestimated. But, in the presence of heterogeneity, difference in terms of coverage probability was observed among the variance estimation methods. Further, wide confidence interval retained higher coverage probability. However, DerSimonian \& Laird and Hunter \& Schmidt methods had coverage probability of 95\% confidence interval around nominal level with precise confidence interval.

\section{Discussion}

It is a global consensus that method of meta-analysis should be guided by the extent of heterogeneity, fixed effect method for lower extent of heterogeneity and random effect method for substantial heterogeneity. ${ }^{13,14}$ But pooled effect size calculated under random effect method also relies on the method used to estimate between study variance. Apart from the non-iterative methods of estimating $\tau^{2}$ discussed in present study, iterative methods, viz. maximum likelihood $\operatorname{method}^{15,16}$, restricted maximum likelihood method ${ }^{17}$, approximate maximum likelihood method ${ }^{3}$, Paule and Mandel's method ${ }^{5,18}$ and Empirical Bayes method ${ }^{15,19}$ also exist. However, these iterative methods involve $\tau^{2}$ itself for calculating $\tau^{2}$. Hence, these methods may not converse all the times. As observed in present study, REM is sensitive to the estimation of between study variance.

Keeping in view of three considered measures of comparing the estimation methods regarding between study variance, Hunter and Schmidt and DerSimonnian \& Laird methods may be preferred. Simulation studies had found that Hunter and Schmidt method produces negatively biased results ${ }^{20}$, but it was not observed in the present study. The pooled effect estimate under REM using these two methods had precise confidence intervals in comparison to other methods. Although coverage probability was found higher under some of the other methods as well, considering precision these two methods with optimal coverage probability are preferred choice.

Among these methods of estimating between study variance, DerSimonian \& Laird Method is widely popular method and available in most of the statistical software facilitating meta-analysis. Although Hunter \& Schmidt method is less popular but it was found to be equally proficient with DerSimonian \& Laird method. Use of these two variance estimation methods over other such methods will provide reliable estimate of various epidemiological questions.

\section{Conflicts of interest}

None.

\section{Funding}

The present research work was not funded by any funding agency.

\section{Author's contribution}

All the three authors conceived \& designed the study. Dr. Mona Pathak did statistical analysis. All authors interpreted the results. The final manuscript of the research work was prepared by Dr. Mona Pathak. All the authors read and provided their inputs to finalize the manuscript.

\section{Author's Information}

Currently, Dr. Mona Pathak is Assistant Professor in Kalinga Institute of Medical Sciences, Bhubaneswar, India. She was Ph.D. student under the supervision of Prof. SN Dwivedi at the time of this research work. Dr. SN Dwivedi is professor in All India Institute of Medical Sciences (AIIMS), New Delhi, India. Dr. Bhaskar Thakur is Assistant Professor in Kalinga Institute of Medical Sciences, Bhubaneswar, India. 


\section{Acknowledgement}

We sincerely acknowledge All India Institute of Medical Sciences, New Delhi for providing resources.

\section{Appendix A. Supplementary data}

Supplementary data to this article can be found online at https:// doi.org/10.1016/j.cegh.2019.06.011.

\section{References}

1. Borenstein M, ed. Introduction to Meta-Analysis. Chichester, U.K: John Wiley \& Sons; 2009:421.

2. Pathak M, Deo SVS, Dwivedi SN, et al. Role of neoadjuvant chemotherapy in breast cancer patients: systematic review and meta-analysis. Indian J Med Pediatr Oncol Print [Internet]. 2019 Jan. [cited 2018 Jul 8]; Available from:. http://www.ijmpo.org/ aheadofprint.asp.

3. Veroniki AA, Jackson D, Viechtbauer W, et al. Methods to estimate the between-study variance and its uncertainty in meta-analysis. Res Synth Methods. 2016 Mar;7(1):55-79.

4. Kacker RN, Datla RU, Parr AC. Statistical analysis of CIPM key comparisons based on the ISO Guide. Metrologia. 2004;41(4):340.

5. DerSimonian R, Kacker R. Random-effects model for meta-analysis of clinical trials: an update. Contemp Clin Trials. 2007 Feb;28(2):105-114.

6. Cochran WG. Some methods for strengthening the common $\chi^{2}$ tests. Biometrics. 1954;10(4):417-451.
7. DerSimonian R, Laird N. Meta-analysis in clinical trials. Contr Clin Trials. 1986 Sep 1;7(3):177-188.

8. Hartung J, Makambi KH. Reducing the number of unjustified significant results in meta-analysis. Commun Stat Simulat Comput. 2003 Jan 11;32(4):1179-1190.

9. Hartung J, Makambi KH. Positive estimation of the between-study variance in metaanalysis: theory and methods. S Afr Stat J. 2002 Jan 1;36(1):55-76.

10. Hunter JE, Schmidt FL. Methods of Meta-Analysis: Correcting Error and Bias in Research Findings. second ed. Thousand Oaks, Calif: Sage; 2004:582.

11. Sidik K, Jonkman JN. A comparison of heterogeneity variance estimators in combining results of studies. Stat Med. 2007 Apr 30;26(9):1964-1981.

12. Sidik K, Jonkman JN. A note on variance estimation in random effects meta-regression. J Biopharm Stat. 2005;15(5):823-838.

13. Tufanaru C, Munn Z, Stephenson M, Aromataris E. Fixed or random effects metaanalysis? Common methodological issues in systematic reviews of effectiveness. Int $J$ Evid Based Healthc. 2015 Sep;13(3):196-207.

14. Hedges LV, Vevea JL. Fixed-and random-effects models in meta-analysis. Psychol Methods. 1998;3(4):486.

15. Thompson SG, Sharp SJ. Explaining heterogeneity in meta-analysis: a comparison of methods. Stat Med. 1999 Oct 30;18(20):2693-2708.

16. Hardy RJ, Thompson SG. A likelihood approach to meta-analysis with random ef fects. Stat Med. 1996 Mar 30;15(6):619-629.

17. Raudenbush SW. Analyzing effect sizes: random-effects models. Handb Res Synth Meta-Anal. 2009:295-315.

18. Paule RC, Mandel J. Consensus values, regressions, and weighting factors. J Res Nat Inst Stand Technol. 1989;94(3):197-203.

19. Morris CN. Parametric empirical Bayes inference: theory and applications. J Am Stat Assoc. 1983 Mar 1;78(381):47-55

20. Viechtbauer W. Bias and efficiency of meta-analytic variance estimators in the random-effects model. J Educ Behav Stat. 2005;30(3):261-293. 